\title{
Study of Swarm Behavior in Modeling and Simulation of Cluster Formation in Nanofluids
}

\author{
Mohammad Pirani, Hassan Basirat Tabrizi, and Ali Farshad \\ Department of Mechanical Engineering, Amirkabir University of Technology, P.O. BOX 15875-4413, Tehran 159163411, Iran \\ Correspondence should be addressed to Hassan Basirat Tabrizi; hbasirat@aut.ac.ir
}

Received 8 April 2012; Revised 4 January 2013; Accepted 10 January 2013

Academic Editor: Azah Mohamed

Copyright (C) 2013 Mohammad Pirani et al. This is an open access article distributed under the Creative Commons Attribution License, which permits unrestricted use, distribution, and reproduction in any medium, provided the original work is properly cited.

\begin{abstract}
Modeling the multiagents cooperative systems inspired from biological self-organized systems in the context of swarm model has been under great considerations especially in the field of the cooperation of multi robots. These models are trying to optimize the behavior of artificial multiagent systems by introducing a consensus, which is a mathematical model between the agents as an intelligence property for each member of the swarm set. The application of this novel approach in the modeling of nonintelligent multi agents systems in the field of cohesion and cluster formation of nanoparticles in nanofluids has been investigated in this study. This goal can be obtained by applying the basic swarm model for agents that are more mechanistic by considering their physical properties such as their mass, diameter, as well as the physical properties of the flow. Clustering in nanofluids is one of the major issues in the study of its effects on heat transfer. Study of the cluster formation dynamics in nanofluids using the swarm model can be useful in controlling the size and formation time of the clusters as well as designing appropriate microchannels, which the nanoparticles are plunged into.
\end{abstract}

\section{Introduction}

Swarming, as a novel approach in modeling the dynamics of multiagent systems, inspires from the behavior of biological self-organized and decentralized systems that cooperate to do a special task. Decentralization means that the swarm has no central leader or boss and each member does its work with a kind of imitation. First attempts to describe the behavior of such biological systems from the mathematical points of view belong to Breder [1] who developed the motion equation of schools of fish and claimed that the motion of each member is the resultant of a long-range attraction and short-range repulsion components. Gazi and Passino [2,3] described the stability of swarm systems.

Many efforts have been investigated to introduce the behavior of multiagent systems whose members have mechanical interaction with each other. It means they may collide to one another and exchange some momentum or arrange in special configurations that can be seen in cluster formations from multiphase and granular flows to nanofluids. A particle dynamic description of solid particles in multiphase flows is one of the aspects of multiagent mechanical systems. Therefore, many attempts were dedicated to describe the behavior of particles in multiphase flows (see Hase [4], Li and Kuipers [5]).

Dorigo et al. [6] introduced an optimization method based on the dynamics of the swarm of ant colonies. The essence of the swarm model is to introduce a consensus between the agents in the form of the summation of attraction and repulsion components between the agents. There have been some efforts to introduce the interactions between the particles as an algebraic summation of the attraction and repulsion coefficients. Sadus [7] proposed an experimental correlation as a potential field for nonpolar particles comprises of attraction and repulsion terms. Zohdi [8] proposed the idea of breaking all of the interactions between particles in a multiphase flow into four major forces, for example, thermal force, drag force, inter particle contact force, and near field force. He modeled the near field force with a linear combination of an attraction and repulsion components just as what is considered in the swarm model. 
Nanofluids are used in many applications because of their specific characteristics. Their capability to enhance the heat transfer is one of those most obvious characteristics. Various kinds of nanoparticles used for these applications such as $\mathrm{Al}_{2} \mathrm{O}_{3}, \mathrm{Fe}_{3} \mathrm{O}_{4}$, and $\mathrm{CuO}$. Increasing the heat transfer depends on the shape and the size of the particles and their volume fraction. Decreasing the size of the nanoparticles causes the conducting heat transfer coefficient to increase [9-13]. Maïga et al. [14] and Chandrasekar et al. [15] also investigated the effect of the host liquid on the heat transfer by comparing water- $\mathrm{Al}_{2} \mathrm{O}_{3}$ and the Glycol- $\mathrm{Al}_{2} \mathrm{O}_{3}$ nanofluids. Brownian motion of the particles and cluster formation in nanofluids are the major issues in the studies of nanofluids according to the important effect of their size on the heat transfer rate. Cluster formation causes the viscosity of nano fluid and thermal conductivity to change [16-19]. Although there have been some efforts in modeling the behavior of nanoparticles in nanofluids, no special research has been done to describe the cohesion and cluster formations of these particles analytically, and most of these works were numerical or experimental approach to this point.

This study proposes a model for cluster formation of the nanoparticles in nanofluids and discusses about their size according to the interparticle forces. Model is based on the swarm model, which enables us to make a better control on the cluster formation phenomenon by knowing the control variables of the system. In addition, this model can be used for analyzing the cohesiveness of nanorobot probes, as nanoparticles injected into the blood vessels [20]. By knowing the cluster size of the swarm set as well as the diameter of the vessel, one can easily compute the number of nano robot probes participating in the cohesion task.

\section{Swarm Model}

A swarm system consists of $N$ members, with mass $m$ for each individual, who are placed in an $n$-dimensional Euclidean space. The motion equation of each member of the swarm in the general form can be written as

$$
\begin{aligned}
& m \ddot{r}_{i}+c \dot{r}_{i} \\
& =\sum_{j=1, j \neq i}^{N}\left(-\frac{\left(r_{i}-r_{j}\right)}{\left|r_{i}-r_{j}\right|}\right)\left(f_{a}\left(\left\|r_{i}-r_{j}\right\|\right)-f_{r}\left(\left\|r_{i}-r_{j}\right\|\right)\right) .
\end{aligned}
$$

In this equation, $f_{a}\left(\left\|r_{i}-r_{j}\right\|\right)$ denotes the attraction between member $i$ with member $j$, and $f_{r}\left(\left\|r_{i}-r_{j}\right\|\right)$ represents the repulsion between the pair $i$ and $j$. Thus the dynamics of member $i$ depend on the resultant of attraction and repulsion forces between $i$ th and other $N-1$ members in the system. Since the attraction and repulsion forces behave like a spring, they are called spring shape forces. Therefore, (1) recalls some generalized form of a mass, spring, and damper oscillator. Coefficient $c$ can be assumed as a damper constant for such a system and is necessary for the stability of the system.
Now define a special class of such attraction and repulsion functions to convert (1) into

$$
\begin{aligned}
& m \ddot{r}_{i}+c \dot{r}_{i} \\
& =\sum_{j=1, j \neq i}^{N}-\frac{\left(r_{i}-r_{j}\right)}{\left|r_{i}-r_{j}\right|}\left(a\left\|r_{i}-r_{j}\right\|^{-\gamma_{1}}-b\left\|r_{i}-r_{j}\right\|^{-\gamma_{2}}\right) .
\end{aligned}
$$

Here $a$ and $b$ are the attraction and repulsion coefficients, respectively. $\gamma_{1}$ and $\gamma_{2}$ are some positive real numbers, and their values depend on the physics of the system which will be described in the following sections. In order to apply this equation for more real mechanical agents such as nanoparticles in nano fluid flows, it is necessary to define some appropriate attraction and repulsion functions well matched with the physics of the problem.

2.1. Adding Diameter to Each Member. In order to induce dimension, or diameter, to the point-shaped members, we must conduct a strategy to inhibit particles to obtain same coordinates in space. In other words, they should not be overlap on each other. Therefore, it is necessary to define a repulsion function that prohibits the distance to be zero or even approaches to zero. Such a repulsion function is called unbounded repulsion [3]. Thus the degree of the repulsion function's denominator, or pole, should be greater than one. Such a function can be defined as

$$
\lim _{\left\|r_{i}-r_{j}\right\| \rightarrow 0^{+}} f_{r}\left(\left\|r_{i}-r_{j}\right\|\right)\left\|r_{i}-r_{j}\right\|=\infty .
$$

This condition inhibits particles to reduce their distance to zero. It can be extended to more general that we can introduce a function that inhibits members to be closer than a particular distance like $C$. This is obtainable with just a modification in the above limit into the following limit:

$$
\lim _{\left\|r_{i}-r_{j}\right\| \rightarrow C} f_{r}\left(\left\|r_{i}-r_{j}\right\|\right)\left\|r_{i}-r_{j}\right\|=\infty \text {. }
$$

$C$ is the distance between the centers of two members or their diameter if they are spherical shaped particles. Therefore, the repulsion function is defined as follows:

$$
f_{r}\left(\left\|r_{i}-r_{j}\right\|\right)=\frac{b}{\left(\left\|r_{i}-r_{j}\right\|-C\right)^{2}} .
$$

Thus, the value of $\gamma_{2}$ in (2) will be equal to 2 .

A long-range attraction function should be defined as well. Since the effects of long-rage attraction function are considerable through the long distances, this function should have direct ratio with the distance between each couple. On the other hand, in realistic swarm systems when two members become too far apart from one another, they lose and forget their effects. Therefore, a relative long-range attraction function should satisfy what follows:

$$
\lim _{\left\|r_{i}-r_{j}\right\| \rightarrow \infty} f_{a}\left(\left\|r_{i}-r_{j}\right\|\right)=0 .
$$


The infinity in above equation is a mathematical infinity. In other words, the physical infinity can be determined according to the application. For example, it can be assumed 100 times longer than the particle's diameter. By defining $l=$ $100 d$ in which $d$ is the agent's diameter, so

$$
\lim _{\left\|r_{i}-r_{j}\right\| \rightarrow l} f_{a}\left(\left\|r_{i}-r_{j}\right\|\right)=0 .
$$

For obtaining this in mind, the basic model that indicates each agent should interact with all other agents. It will be modified into the case; each agent interacts with some agents in its neighborhood, which is according to the sensitivity of the agent for receiving the long-range attraction signals.

Therefore, the attraction function can be defined as

$$
f_{a}\left(\left\|r_{i}-r_{j}\right\|\right)=\frac{a}{\left\|r_{i}-r_{j}\right\|}
$$

and the value of $\gamma_{1}$ in (2) will be equal to 1 .

It is worth to mention that this attraction function is called relative attraction function for long-range relative to the repulsion function. According to (8), it not only has direct ratio with distance, but also has an inverse ratio with it. Nevertheless, this inverse ratio is in the first power. Thus, it can be concluded this attraction function is long range relative to the repulsion that has an inverse ratio with the second power of distance.

2.2. Adding Environmental Effects. In common swarm models, the environmental effects are considered as well. These effects are modeled as a profile that can attract or repel members. In fact, this profile is considered as a simulation of nutrient that attracts member to itself or as a model of toxin, which repels members far apart. However, it can be interpreted in a more general case as a potential field exerting on each member from the environment. The motion of individuals will be through the opposite direction of the profile's gradient.

In order to add this effect into our model, we considered a potential function like $g(\cdot): R^{n} \rightarrow R$ and rewrite (1) as follows:

$$
\dot{r}_{i}=-\nabla_{r_{i}} g\left(r_{i}\right)+\sum_{j=1, j \neq i}^{N} f\left(r_{i}-r_{j}\right) .
$$

The ambient profile function can be interpreted in many kinds. In this paper, we use the plane profile as

$$
g(r)=A^{T} r+b .
$$

Then the equation of motion regarding the environmental effect will be

$$
\begin{aligned}
\ddot{r}^{i}+ & \frac{1}{m_{i}} \dot{r}^{i} \\
= & \frac{1}{m_{i}}\left(\sum_{j=1, j \neq i}^{N}-\frac{\left(r_{i}-r_{j}\right)}{\left|r_{i}-r_{j}\right|}\left(\frac{a}{\left\|r_{i}-r_{j}\right\|}-\frac{b}{\left(\left\|r_{i}-r_{j}\right\|-C\right)^{2}}\right)\right) \\
& -\nabla_{r_{i}} g\left(r_{i}\right) .
\end{aligned}
$$

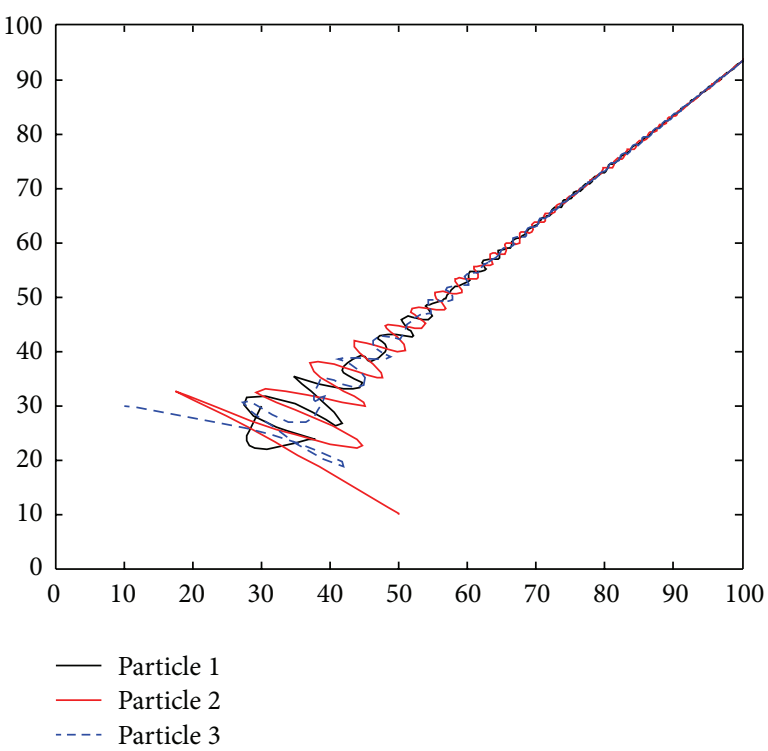

FIGURE 1: Two-dimensional simulation of (11) for three particles, $m_{i}=2, a=100, b=10$, and $\nabla_{r_{i}} g\left(r_{i}\right)=2$.

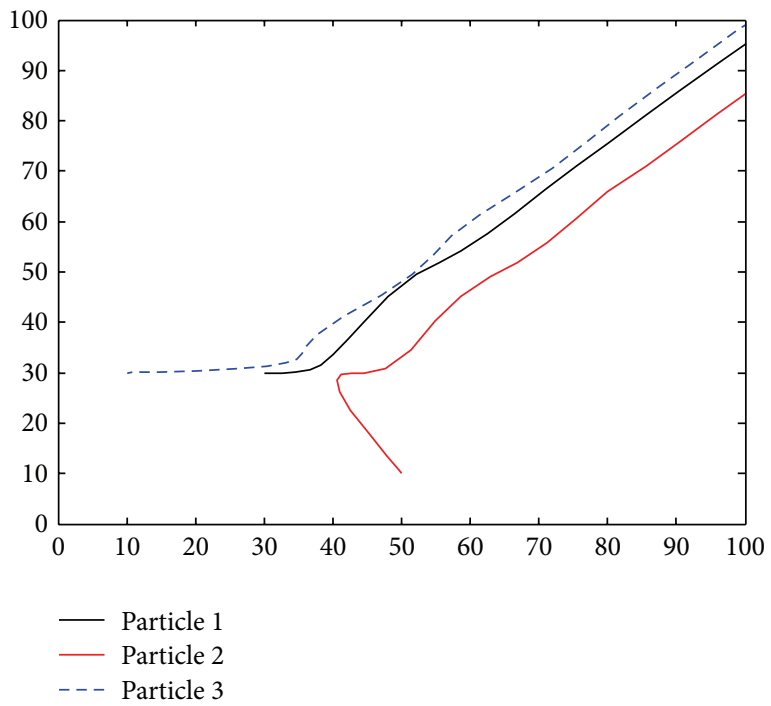

FIgURE 2: Two-dimensional simulation of (11) for three particles, $m_{i}=2, a=10, b=100$, and $\nabla_{r_{i}} g\left(r_{i}\right)=2$.

Two simulation examples are provided in Figures 1 and 2. Consider the effect of the attraction and the repulsion coefficients in the convergence radius of the particles. In both cases the plane profile with $\nabla g(r)=2$ is assumed.

\section{Dimensional Analysis}

In order to analyze (1) and apply it for our special case, it is necessary to make a physical sense about each term in that equation. For simplicity and without losing the generality, 
assume the swarm system consists of just two members. Therefore, the sigma sign in (1) disappears, and we have

$$
m \ddot{r}+d \dot{r}=a r^{-\gamma_{1}}-b r^{-\gamma_{2}} .
$$

Equation (12) is a nonlinear ordinary differential equation. Constants $\gamma_{1}$ and $\gamma_{2}$ can be determined according to our desire in choosing any kind of attraction and repulsion components.

It is time to apply physical properties of the system of nanoparticles in the nano fluid, which can be considered as nanorobot probes in the blood. The viscosity of blood is ten times higher than water, and we can claim that the effect of mass of each robot is negligible. To prove this claim, refer to experimental and more realistic approaches. Cavalcanti et al. [20] investigated in an experiment $10^{12}$ nanorobots with the total mass of just 0.2 gram in 5 lit bloods of a typical adult $[21,22]$.

Since, the ratio of inertia force to the viscous force is a dimensionless number called the Reynolds number, which is

$$
\text { Reynolds }=\frac{d \rho v}{\mu} .
$$

Here $d, \rho, v$, and $\mu$ are the diameter of the particle, the density of the fluid, the average velocity of the flow, and the dynamic viscosity of the flow, respectively.

The velocity of the flow in a small blood vessel is assumed about $1 \mathrm{~mm} / \mathrm{s}$. The value for the density and viscosity of blood plasma is

$$
\rho=1 \mathrm{gr} / \mathrm{cm}^{3}, \quad \mu=10^{-2} \mathrm{gr} / \mathrm{cm} \cdot \mathrm{s} .
$$

According to the above values, the Reynolds number for a nanorobot with the diameter of $1 \mu \mathrm{m}$ is

$$
\text { Reynolds }=\frac{d \rho v}{\mu} \approx 10^{-3} .
$$

For the case of water- $\mathrm{Al}_{2} \mathrm{O}_{3}$, nanofluids the Reynold's number value is about $10^{-4}$. This Reynolds number indicates that the viscosity of the host liquid is at least $O\left(10^{3}\right)$ higher than the mass of the particles.

Consider a dimensionless time $\tau$ as proposed in [23]

$$
\tau=\frac{t}{T}
$$

A new derivative with respect to $\tau$ follows:

$$
\begin{gathered}
\dot{r}=\frac{d r}{d t}=\frac{d r}{d \tau} \frac{d \tau}{d t}=\frac{1}{T} \frac{d r}{d \tau}, \\
\ddot{r}=\frac{d^{2} r}{d t^{2}}=\frac{d}{d t}\left(\frac{1}{T} \frac{d r}{d \tau}\right)=\frac{1}{T^{2}} \frac{d^{2} r}{d \tau^{2}} .
\end{gathered}
$$

Substituting above relations into (12) gives

$$
\frac{m}{T^{2}} \frac{d^{2} r}{d \tau^{2}}+\frac{c}{T} \frac{d r}{d \tau}=a r^{-\gamma_{1}}-b r^{-\gamma_{2}}
$$

Dividing both sides by $m$ yields

$$
\frac{1}{T^{2}} \frac{d^{2} r}{d \tau^{2}}+\frac{c}{m T} \frac{d r}{d \tau}=\frac{a}{m} r^{-\gamma_{1}}-\frac{b}{m} r^{-\gamma_{2}} .
$$

Now choose the value of $T$ in order to satisfy the following condition:

$$
\frac{c}{m T}=o(1)
$$

An appropriate choice for $T$ can be $T=c / m$.

So

$$
\frac{1}{T^{2}} \ll 1 \text { yields } \frac{m^{2}}{c^{2}} \ll 1 \Longrightarrow m^{2} \ll c^{2} \text {. }
$$

This can be interpreted as expressing that the damping coefficient in (12), which is the viscosity of the fluid, is very strong, or the mass is very small. It exactly has the same meaning with the small Reynolds number, which was mentioned above.

In a precise sense define $\varepsilon$, such that

$$
\varepsilon=\frac{m^{2}}{c^{2}} .
$$

Thus, (19) becomes

$$
\varepsilon \frac{d^{2} r}{d \tau^{2}}=-\frac{d r}{d \tau}+\frac{a}{m} r^{-\gamma_{1}}-\frac{b}{m} r^{-\gamma_{2}} .
$$

The character $\varepsilon$ is too small, so one can eliminate the right hand side as follows:

$$
\frac{d r}{d \tau}=\frac{a}{m} r^{-\gamma_{1}}-\frac{b}{m} r^{-\gamma_{2}}=f(r) .
$$

The main question that naturally arises is that how much precise (24) can be and in what circumstances one can use it as a description of the system. To answer this question, we analyzed the behavior of (23) in the phase space. By defining a new parameter $R,(23)$ will be

$$
\begin{gathered}
\frac{d r}{d \tau}=R \\
\varepsilon \frac{d R}{d \tau}=-R+\frac{a}{m} r^{-\gamma_{1}}-\frac{b}{m} r^{-\gamma_{2}} .
\end{gathered}
$$

By defining, $f(r)=(a / m) r^{-\gamma_{1}}-(b / m) r^{-\gamma_{2}}$ turns into

$$
\begin{gathered}
\frac{d r}{d \tau}=R, \\
\frac{d R}{d \tau}=\frac{1}{\varepsilon}(f(r)-R) .
\end{gathered}
$$

By considering that the phase plane portraits this system and curve $C: f(r)-R=0$ in this plane. This curve demonstrates the first-order system because the second equation in (26) equals to zero. We claim that the actual second-order system will converge to this curve as time progress. For proving, consider the actual curve starts from an $O(1)$ distance bellow 
the curve $C$. According to the second equation in (26), one has $d R / d \tau=O(1 / \varepsilon)$. Therefore, the curve suddenly jumps into $f(r)-R=O(\varepsilon)$, and as $\varepsilon \rightarrow 0$ this region will be indistinguishable with $C$.

From the above discussion, one can conclude that the system in (26) can behave like a first order system but after a time lag $T$. Before this time lag, we are not allowed to eliminate the second-order derivative. In other words, the first order system behaves with a desired precision at $t>T$. This is exactly what one expects from the system. Since, the desired property of this system is its convergence radius as time approaching to infinity.

Thus, according to above analysis on (26) in the phase space, we can claim that (23) can be reduced into (24) as follows:

$$
\frac{d r}{d \tau}=\frac{a}{m} r^{-\gamma_{1}}-\frac{b}{m} r^{-\gamma_{2}}
$$

and in general form for many agents we have

$$
\dot{r}_{i}=\sum_{j=1, j \neq i}^{N}-\frac{\left(r_{i}-r_{j}\right)}{\left|r_{i}-r_{j}\right|}\left(\frac{a}{m}\left\|r_{i}-r_{j}\right\|^{-\gamma_{1}}-\frac{b}{m}\left\|r_{i}-r_{j}\right\|^{-\gamma_{2}}\right) .
$$

The convergence radius can be easily determined from (28). For example, for the repulsion and attraction functions which were introduced in (5) and (8), respectively, for the first order (28), Gazi and Passino [2] determined the maximum convergence radius is not greater than $b / a$. This bound is for the swarm set whose agents have no diameter. In addition, this bound is independent to the number of the agents. To include the volume of each agent and the number of the agents participating in the cohesion task, one need to modify this bound. To yield this, consider $N$ swarm members, which are accumulating in 3-dimensional spaces. If all the agents stick together to form a cluster, the swarm volume size will be

$$
\text { The swarm volume size }=\frac{4}{3} \pi C^{3} N \text {. }
$$

If all these agents converge to a sphere, the minimum radius of such a sphere can be determined easily by equaling the accumulated swarm volume and the volume of the sphere as follows:

$$
\frac{4}{3} \pi C^{3} N<\frac{4}{3} \pi r_{\min }^{3} \Longrightarrow r_{\min }>C \sqrt[3]{N}
$$

So the previous bound modifies to

$$
\text { The accurate size }=\frac{b}{a} C \sqrt[3]{N}
$$

\section{A Design Problem: Finding the Number of Participating Nanorobot Probes}

Consider a group of nanorobots in a blood vessel cooperating for a special purpose that can be finding in some cancerous cells. According to experimental notes, $10^{12}$ robots are injected through the veins [20]. A typical adult has 5 lit bloods. Therefore, the density of nano robots becomes $2 \times 10^{-4}$ nanorobots $/ \mu \mathrm{m}^{3}$.

The maximum convergence radius for a swarm system with these attraction and repulsion functions in (5) and (8) for maintaining the density constant according to (31) is

$$
\varepsilon<\frac{b}{a} C \sqrt[3]{N}
$$

Therefore, the density of nanorobots is

$$
\text { density }=\frac{N}{(4 / 3) \pi \varepsilon^{3}}=\frac{N}{(4 / 3) \pi(C \sqrt[3]{N} b / a)^{3}}=\frac{3 a^{3}}{4 \pi b^{3} C^{3}} .
$$

Equaling this formula with the experimental measured density [20] of cooperative nanorobots in blood vessels gives

$$
\frac{3 a^{3}}{4 \pi b^{3} C^{3}}=2 \times 10^{-4} \text { Robot } / \mu \mathrm{m}^{3} .
$$

Considering $1 \mu \mathrm{m}$ diameter for each agent follows

$$
\left(\frac{a}{b}\right)^{3}=2 \times 10^{-4} \times 4 \pi \times \frac{C^{3}}{3}=8.38 \times 10^{-4} .
$$

Then the ratio of attraction and repulsion coefficients is

$$
\frac{a}{b}=0.094 \approx 0.1 \text {. }
$$

One of the important aspects of maximum convergence radius in (31) is to determine the number of nanorobot agents, which can accumulate in a vessel with respect to the diameter of the vessel. For instance, suppose that the diameter of nanorobot cohesion cannot exceed a quarter of the diameter of the vein. Hence one can easily calculate the maximum number of robots participate in cohesion. Now assume the diameter of the vein is $D=800 \mu \mathrm{m}$, then

$$
\varepsilon_{\max }=\frac{C \sqrt[3]{N} b}{a} \Longrightarrow d_{\max }=\frac{2 C \sqrt[3]{N} b}{a}=\frac{D}{4} .
$$

As we derived a reasonable ratio of attraction and repulsion constants for nano robots in blood, it yields

$$
\begin{aligned}
\frac{a}{b}=0.1 \Longrightarrow \frac{b}{a}=10, \quad D=800 \mu \mathrm{m}, \quad C=1 \mu \mathrm{m} \\
d_{\max }=\frac{2 C \sqrt[3]{N} b}{a}=20 C \sqrt[3]{N}=\frac{D}{4}=200 \mu \mathrm{m} \Longrightarrow C \sqrt[3]{N} \\
=10 \Longrightarrow N=1000 \text { Robots. }
\end{aligned}
$$

It means that if the robot cohesion is forced to have a diameter less than a quarter of a vein diameter, the maximum numbers of agents that can participate in the cohesion are 1000 nanorobots.

This example could be converted into a design problem of micro channels if it asks the diameter of the vessel by giving the number of participating nanorobots. 


\section{Conclusion}

In this study we discussed about the behavior dynamics of nanoscale grains and particles moving in a host liquid. According to this model, the dynamics of each particle depend on the resultant forces between it and other particles in its neighborhood that are in the form of attraction and repulsion components. One of the most important results of this model was to derive a convergence radius for particulate clustering in nanofluids that is one of the most important phenomena in the field of the researches in micro- and nanofluids especially in the context of heat transfer.

\section{Acknowledgments}

The authors would like to thank Milad Rakhsha from Amirkabir University of Technology, Iran, for bringing some useful references in the field of nanofluidics. Also, thanks are to professor Michael Zavlanos from Duke University, NC, USA, for his helpful comments.

\section{References}

[1] C. M. Breder, "Equations descriptive of fish schools and other animal aggregations," Ecology, vol. 35, pp. 361-370, 1954.

[2] V. Gazi and K. M. Passino, Swarm Stability and Optimization, Springer, New York, NY, USA, 2011.

[3] V. Gazi and K. M. Passino, "A class of attractions/repulsion functions for stable swarm aggregations," International Journal of Control, vol. 77, no. 18, pp. 1567-1579, 2004.

[4] W. L. Hase, "Molecular dynamics of clusters, surfaces, liquids \& interfaces," in Advances in Classical Trajectory Methods, vol. 4, JAI press, 1999.

[5] J. Li and J. A. M. Kuipers, "Gas-particle interactions in dense gas-fluidized beds," Chemical Engineering Science, vol. 58, no. 3-6, pp. 711-718, 2003.

[6] M. Dorigo, V. Maniezzo, and A. Colorni, "Ant system: optimization by a colony of cooperating agents," IEEE Transactions on Systems, Man, and Cybernetics B, vol. 26, no. 1, pp. 29-41, 1996.

[7] R. J. Sadus, Molecular Simulation of Fluids: Theory, Algorithms and Object-Orientation),, Elsevier, New York, NY, USA, 1999.

[8] T. I. Zohdi, "Particle collision and adhesion under the influence of near-fields," Journal of Mechanics of Materials and Structures, vol. 2, no. 6, pp. 1011-1018, 2007.

[9] K. B. Anoop, T. Sundararajan, and S. K. Das, "Effect of particle size on the convective heat transfer in nanofluid in the developing region," International Journal of Heat and Mass Transfer, vol. 52, no. 9-10, pp. 2189-2195, 2009.

[10] H. Chang and Y. C. Chang, "Fabrication of $\mathrm{Al}_{2} \mathrm{O}_{3}$ nano fluid by a plasma arc nano particles synthesis system," Journal of Materials Processing Technology, vol. 207, no. 1-3, pp. 193-199, 2008.

[11] H. Chen, Y. Ding, and A. Lapkin, "Rheological behaviour of nanofluids containing tube / rod-like nanoparticles," Powder Technology, vol. 194, no. 1-2, pp. 132-141, 2009.

[12] W. Lu and Q. Fan, "Study for the particle's scale effect on some thermo physical properties of nano fluids by a simplified molecular dynamics method," Engineering Analysis with Boundary Elements, vol. 32, no. 4, pp. 282-289, 2008.

[13] P. K. Namburu, D. K. Das, K. M. Tanguturi, and R. S. Vajjha, "Numerical study of turbulent flow and heat transfer characteristics of nanofluids considering variable properties," International Journal of Thermal Sciences, vol. 48, no. 2, pp. 290302, 2009.

[14] S. E. B. Maiga, S. J. Palm, C. T. Nguyen, G. Roy, and N. Galanis, "Heat transfer enhancement by using nanofluids in forced convection flows," International Journal of Heat and Fluid Flow, vol. 26, no. 4, pp. 530-546, 2005.

[15] M. Chandrasekar, S. Suresh, and A. Chandra Bose, "Experimental investigations and theoretical determination of thermal conductivity and viscosity of $\mathrm{Al}_{2} \mathrm{O}_{3}$ /water nanofluid," Experimental Thermal and Fluid Science, vol. 34, no. 2, pp. 210-216, 2010.

[16] M. Chopkar, S. Kumar, D. R. Bhandari, P. K. Das, and I. Manna, "Development and characterization of $\mathrm{Al}_{2} \mathrm{Cu}$ and $\mathrm{Ag}_{2} \mathrm{Al}$ nanoparticle dispersed water and ethylene glycol based nanofluid," Materials Science and Engineering B, vol. 139, no. 23, pp. 141-148, 2007.

[17] B. Ghasemi and S. M. Aminossadati, "Brownian motion of nanoparticles in a triangular enclosure with natural convection," International Journal of Thermal Sciences, vol. 49, no. 6, pp. 931-940, 2010.

[18] W. Jiang, G. Ding, H. Peng, and H. Hu, "Modeling of nanoparticles' aggregation and sedimentation in nanofluid," Current Applied Physics, vol. 10, no. 3, pp. 934-941, 2010.

[19] N. R. Karthikeyan, J. Philip, and B. Raj, "Effect of clustering on the thermal conductivity of nano fluids," Materials Chemistry and Physics, vol. 109, no. 1, pp. 50-55, 2008.

[20] A. Cavalcanti, T. Hogg, B. Shirinzadeh, and H. C. Liaw, "Nanorobot communication techniques: a comprehensive tutorial," in Proceedings of the 9th International Conference on Control, Automation, Robotics and Vision (ICARCV'06), Singapore, December 2006.

[21] R. A. Freitas Jr., Basic Capabilities, vol. 1 of Nanomedicine, Landes Bioscience, Georgetown, Tex, USA, 1999.

[22] R. A. Freitas Jr., Biocompatibility, vol. 2A of Nanomedicine, Landes Bioscience, Georgetown, Tex, USA, 2003.

[23] S. H. Strogatz, Nonlinear Dynamic and Chaos: With Applications to Physics, Biology, Chemistry and Engineering, Addison-Wesley, Reading, Mass, USA, 1994. 

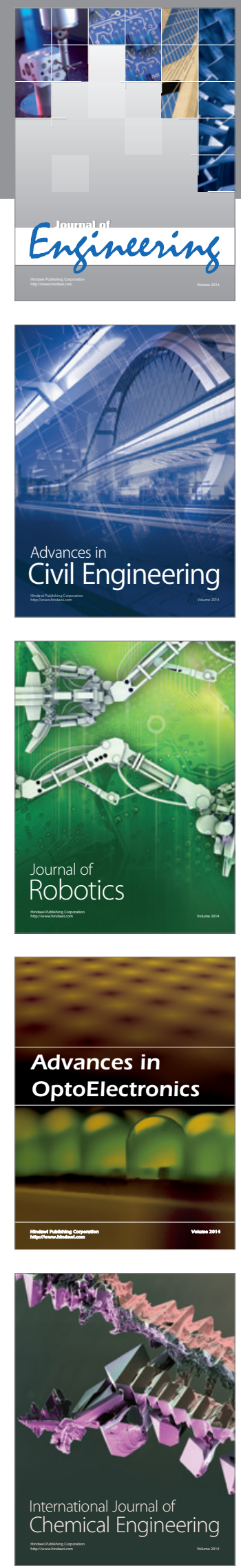

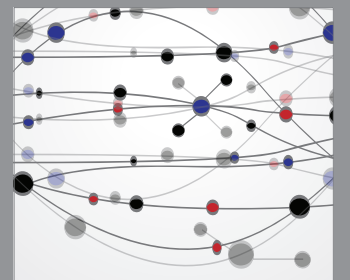

The Scientific World Journal
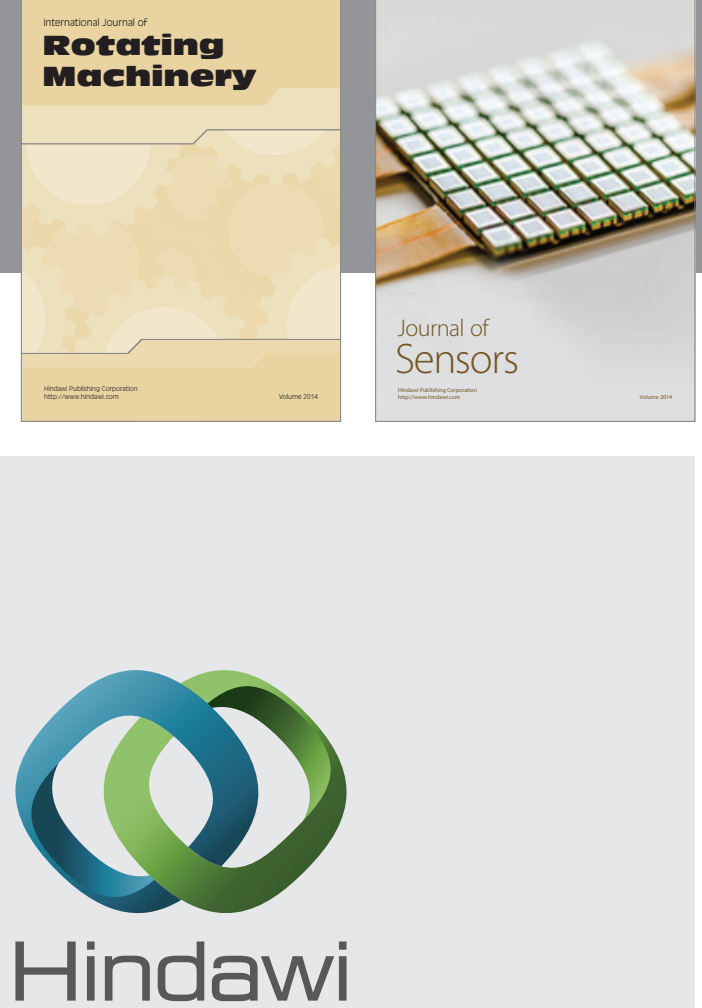

Submit your manuscripts at http://www.hindawi.com
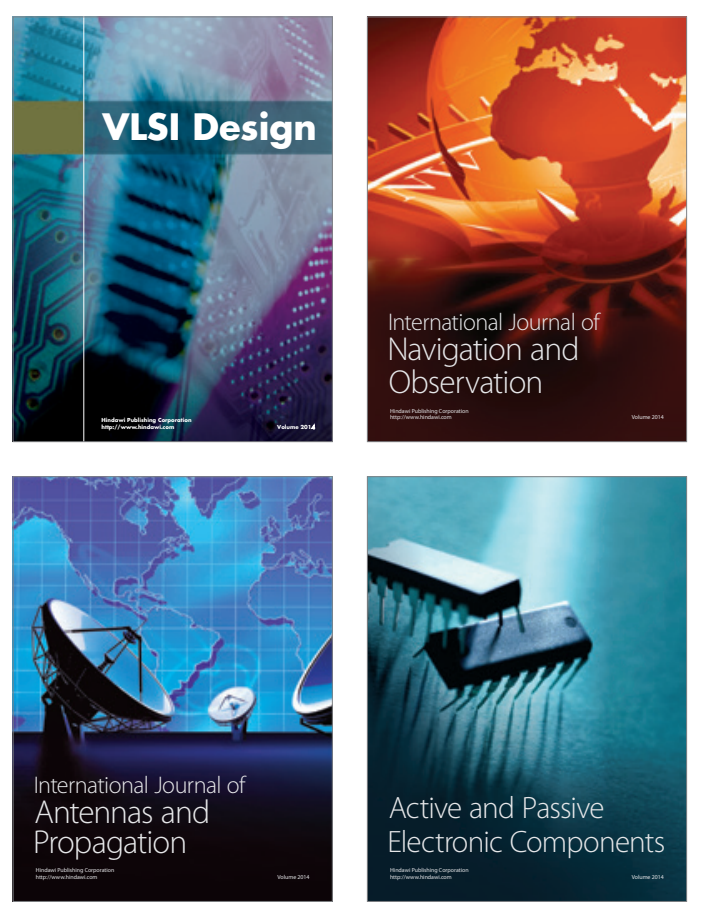
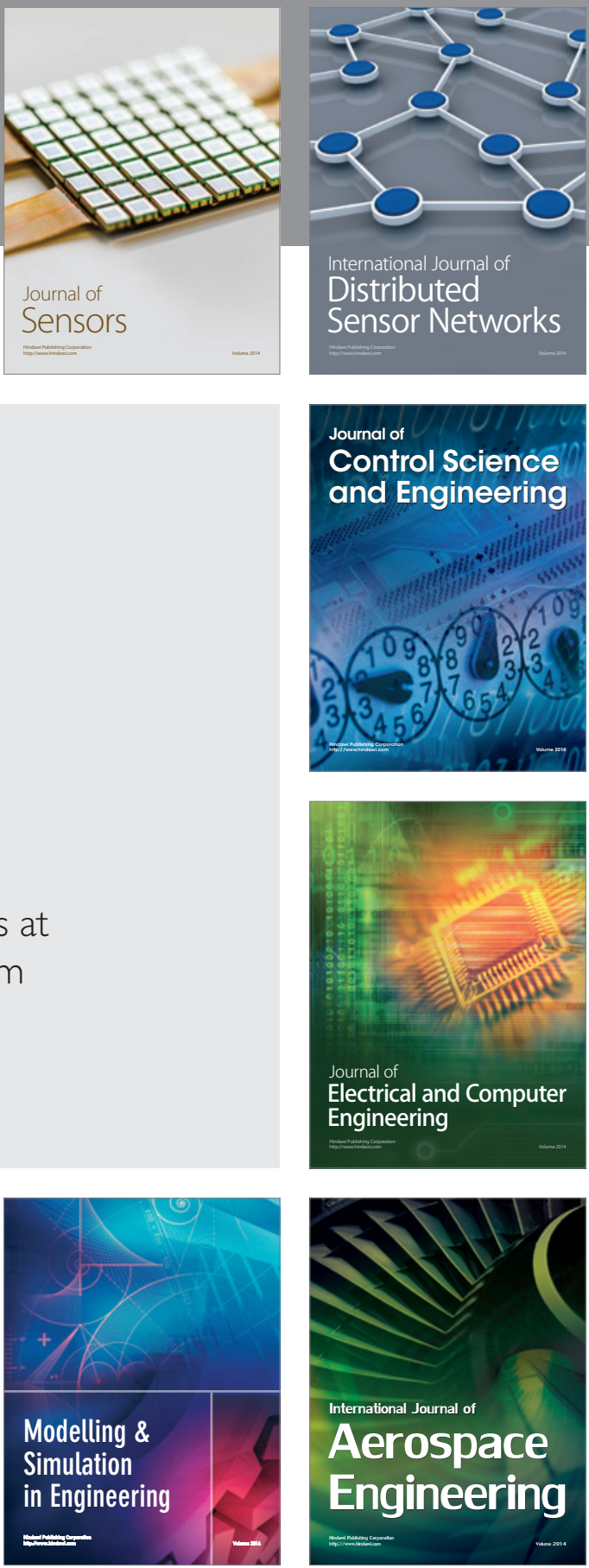

Journal of

Control Science

and Engineering
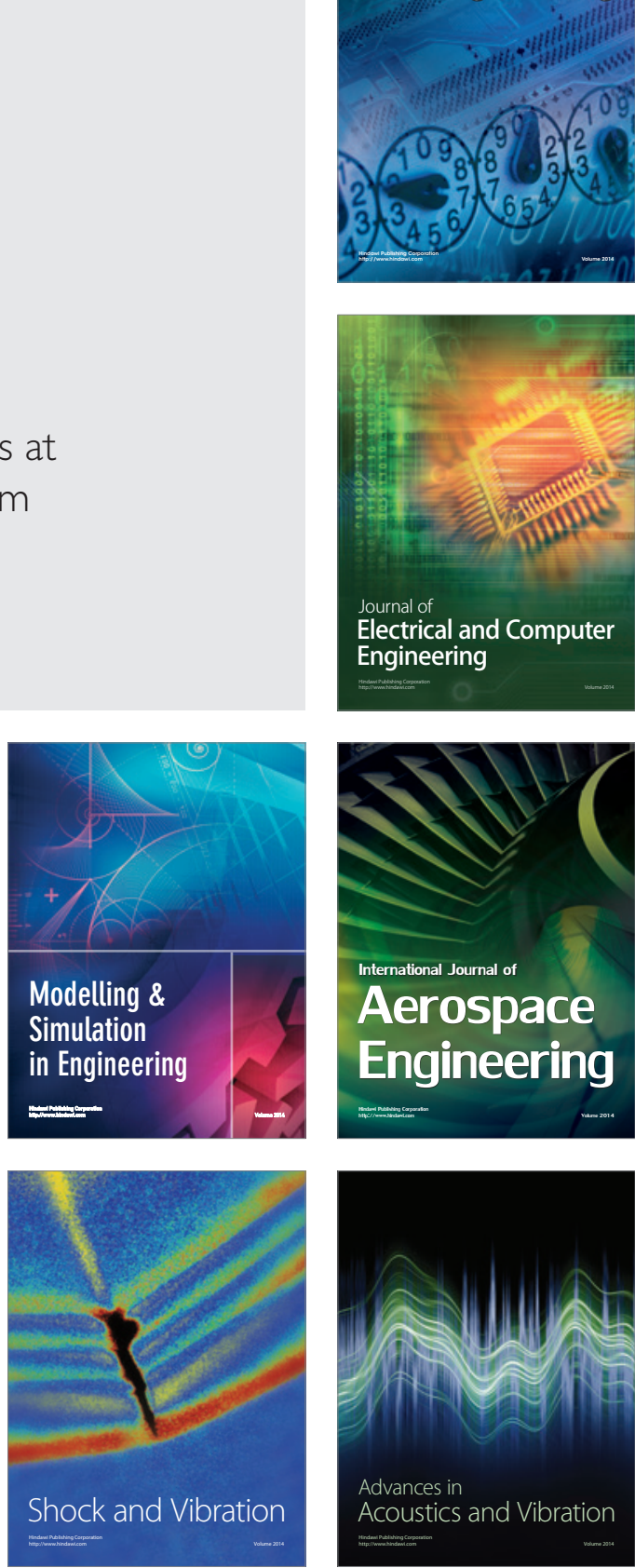